\title{
Numerical Study of Plasmonic Effects of Ag Nanoparticles embedded in the Active Layer on performance polymer organic Solar Cells
}

Leila Shabani

Persian Gulf University

ahmad mohammadi ( $\nabla$ mohammadi@pgu.ac.ir)

Persian Gulf University https://orcid.org/0000-0002-2472-9897

Tahmineh Jalali

Persian Gulf University

\section{Research Article}

Keywords: Absorption, Finite Difference Time Method, plasmonic Nanoparticles, Organic Solar Cells

Posted Date: July 29th, 2021

DOl: https://doi.org/10.21203/rs.3.rs-551348/v1

License: (c) (i) This work is licensed under a Creative Commons Attribution 4.0 International License.

Read Full License

Version of Record: A version of this preprint was published at Plasmonics on September 29th, 2021. See the published version at https://doi.org/10.1007/s11468-021-01539-x. 


\title{
Numerical Study of Plasmonic Effects of Ag Nanoparticles embedded in the Active Layer on performance polymer organic Solar Cells
}

\author{
Leila Shabani, Ahmad Mohammadi (Corresponding author), Tahmineh Jalali \\ Physics Department, Persian Gulf University, Bushehr 7516913718, Iran \\ Tel: +98-771-334-54408 E-mail: mohammadi@pgu.ac.ir
}

\begin{abstract}
In this paper, the light absorption the active layer of polymer polymer solar cells (OPV) by using plasmonic nanocrystals with hexagonal lattice is investigated. To study the relation between the performance of the OPV solar cell and its active layer, a three-dimensional model for its morphology is utilized. Therefore, the threedimensional (3D) finite-difference time-domain method and Lumirical software were used to measure the field distribution and light absorption in the active layer in terms of wavelength. OPV solar cells with bilayer and bulk heterojunction structured cells were designed using hexagonal lattice crystals with plasmonic nanoparticles, as well as, core-shell geometry to govern a design to optimize light trapping in the active layer. The parameters of shape, material, periodicity, size, the thickness of the active layer as a function of wavelength in OPV solar cells have been investigated. A very thin active layer and an ultra-thin shell were used to achieve the highest increase in optical absorption. The strong alternating electromagnetic field around the core-shell plasmonic nanoparticles resulting from the localized surface plasmon resonance (LSPR) suggested by the Ag plasmonic nanocrystals increased the intrinsic optical absorption in the active layer poly(3-hexylthiophene):phenyl-C61butyric acid methyl ester (P3HT:PCBM). Based on the photovoltaic results the short circuit current ranged from 19.7 to $26.7 \mathrm{~mA} / \mathrm{cm} 2$.
\end{abstract}

Keywords: Absorption, Finite Difference Time Method, plasmonic Nanoparticles, Organic Solar Cells

PACs Number: 88.40.hj, 88.40.jj, 42.70.Qs 


\section{Introduction}

In the last decade, organic photovoltaic cells (OPVs) have been attracted great attention, due to their promising potential for the next-generation solar cells because they are lightweight, flexible and low-cost, semi-transparency, solution process ability and large surface area [1]. Therefore, OPV solar cells are capable of alternating with conventional photovoltaic devices, since they reduce the cost of energy consumption. Photovoltaic materials are fabricated in thin-film form to decrease the production cost. Various approaches have been employed to enhance light harvesting in the films. Nevertheless, the absorption of light, especially at a long wavelength, is rather low in the OPV solar cells, since they have the thin active layer thickness and indirect bandgap of polymers. consequently, this prevents the cell absorbing low energy photons in the solar energy spectrum [1-2]. Several light trapping and photon management methods have been suggested to improve the performance of OPV solar cells, to improve the collection efficiency of incident light inside thin-films such as antireflection coating [3-4], photonic crystals [5-6], plasmonic excitation using metallic nanoparticles [7], optimization of the electromagnetic field distribution and periodic metallic grating [1,8-9] which are traditionally based on geometrical optics. The efficiency of these polymer cells is low compared to other cells [1], and this is a significant barrier to widespread commercialization of them.

The efficiency of OPV solar cells has been increased by more than $13-14 \%[4,10]$. In OPVs materials, owing to their short diffusion length in the organic semiconductor materials [11], while fairly low carrier mobility $\left(\sim 10^{4} \mathrm{~cm}^{2} / \mathrm{V}_{\mathrm{s}}\right)$, as well as the photoactive layer is generally rather thin, less than $100 \mathrm{~nm}$ to facilitate the charge carrier diffusion and light extraction [10] is achieved. Even though much photon energy is lost, because of the poor absorption of the incident light [11], numerous researches have been done to further light absorption in the OPVs.

Among these approaches, the ideas of plasmonic metallic nanoparticles and gratings play an important role in absorption enhancement. Different kinds of light scattering methods, from random to periodic plasmonic nanocrystals, at the front and/or backside of the active layer, have utilized which light is diffracted, refracted, or reflected back to increase the total path length of incident light inside the active layer. 
However, placing metallic plasmonic nanocrystal on top of the active layer would block a huge amount of total incident solar power. Currently, using multiple plasmonic nanocrystal to enhance absorption, on top or/and at the bottom of the active layer, has attracted much attention because of the broadband absorption enhancement, as well as less sensitivity to the angle of incident light [12-13]. Metallic nanoparticles have been exploited to increase the photocurrent, as well as efficiency by $33 \%$ of thin-film silicon solar cells [15]. The advantages of the metallic nanoparticles in OPVs have been investigated since they can be used in the device architecture without any significant effort.

Localized surface plasmons (LSP), which are collective oscillations of free electrons in metallic nanoparticles in response to an incident field [3,5], are a type of plasmon, have extensively attracted researchers' attention for different field-enhanced spectroscopy, Photovoltaic and sensing applications [10,7]. In regulation, the light excitation, a resonance, which is named Plasmonic, happens as soon as the frequency of the incident photons reaches the frequency of collective oscillation of conduction electrons of metallic particles, and can interact strongly with external electromagnetic waves [16]. Currently, surface plasmon has become a significant light trapping technique in solar cells [17]. Owing to the near-field nature of the surface plasmons, they are essentially appropriate for application in thin-film devices. Recently, Photonic crystals have also been applied in OPV devices, the same as inorganic solar cells [18-19]. However, this method will be restricted by the relatively low refractive indices of the organic materials since the proper guided modes in photonic crystal devices can be achieved by high index contrast as well as a reasonably thick layer [16-18]. Hence, plasmonic metallic nanoparticles are more appropriate for application in OPV devices [20]. Metallic nanoparticles could enhance scattering and local field from the excitation of localized surface plasmon resonances to improve the OPV performance. Patterned electrodes could enhance local field as well as optical path length by coupling into the surface plasmons to improve the OPV performance. Recently, many papers on plasmonic nanoparticles have been published with applications in OPV technology. The objective of our paper is to use localized plasmons excitation of metallic nanoparticles to trap or confine light inside the photoactive material layer, or to acquire the resonance of the internal light scattering on these plasmonic metallic nanoparticles. This excitation of localized plasmons depends on several parameters, including size, material, the 
surface density of the metallic nanoparticles, as well as wavelength, polarization, and incident angle of light. Finally, light absorption could be enhanced, especially in the desired spectral ranges, where the photovoltaic material absorbs the light weakly. For small periods less than $300 \mathrm{~nm}$, the reason for improving of the light is mostly LSP $[16,19]$. The large grating periods could scatter light into surface plasmons [19, 21-22].

The report on performance enhancement through the combination of gold plasmonic nanoparticles in the Poly(3,4-ethylenedioxythiophene): poly (styrenesulfonate) (PEDOT-PSS) active layer has been presented by Chen et al [23] and a $20 \%$ improvement has been observed. Ion, et. al. achieved an enhancement in photocurrent due to LSPR generation by embedding the layer of Ag nanosphere between PEDOT-PSS and the active layer P3HT-PCBM of the OPV solar cell [24]. Kulkarni et al. obtained three times more generation of charge carrier by a thin layer of Ag material is inserted at the bottom of the active layer [25]. Zhu et al. reported on the 1.40 times absorption enhancement by plasmonic nanostructures the hybrid active layer and optimizing the fabricated structure [21]. Bai et al. published the first reports on a combination of 2D nanotubes on OPV solar cells, resulting in effective polarization-independent absorption [26]. Diukman et al. showed an improvement in the external quantum efficiency of OPV solar cells after embedding $\mathrm{Au}$ nanoparticles arrays in the active layer [27]. Kekeli et al. reported an enhancement in intrinsic optical absorption in the active layer, with and without the square lattice of core-shell (Ag@Sio2) by 24.7\% [22].

The $\mathrm{Au}$ nanoparticles, which are on the order of 5-50 nm, act as antennas, accumulating incident light energy in localized surface plasmon modes with up to a 100 -fold increase in electric field. The generation of a strong near field distribution around the gold nanoparticles embedded in the active layer was simulated and gained an enhancement on the light absorption [17]. These plasmonic nanostructures immersed in the active layer can improve the plasmon and exciton coupling, as well as the generation of hot excitons This generation can enhance the free charges [2830].

In this paper, to increase the efficiency of polymer solar cells using surface plasmon, factors such as the size and material of plasmonic nanocrystals and the effect of these factors on parameters such as absorption and intensity distribution of electromagnetic fields in the active layer of polymer solar cells are investigated.

In section 2, the theoretical and modelling method, as well as simulation layout, is studied in detail. In the third section, the optical effects of plasmonic nanoparticles 
in OPV solar cells are investigated, including: Various morphology size and material of this plasmonic nanocrystal in the active layer of polymer solar cell with bulk heterojunction. The effects of plasmonic nanocrystals on the efficiency of the OPV solar cell by trapping the incident light into the active layer is investigated. Tuning the morphology of a plasmonic nanostructure to achieve higher efficiency is one of the key issues in our research.

\section{Theoretical and Simulation Methods}

\section{1 metallic nanoparticles}

Metallic nanoparticles are investigated as plasmonic structures since the LSPR could be tuned by their physical properties such as size, material and shape. These nanostructures can increase the light absorption by cumulative forward scattering cross-section, as well as near-field light enhancement. When the size of the metallic nanoparticles is equal to or smaller than the incident light wavelength, a strong interaction happens among the electromagnetic field with the free conduction electrons in the metallic nanoparticles [31].

Surface plasmon resonance is the collective oscillations in the electron density at the metal surface due to their interaction with the incident light. The resonance happens when the light coupled to the electrons oscillating at the same wavelength, referred to as LSPR in nanostructures and results in a strong enhancement of the localized electromagnetic field. Surface Plasmons, which are coupled with photons, can excite a collective of conduction electrons to propagate along with an interface between a metallic nanostructure and a dielectric with $\boldsymbol{\varepsilon}_{\boldsymbol{d}}>0$ [31]. The LSP at the vicinity of the interface can be propagated along the metal surface until the energy dissipates. Metallic nanoparticles with a small amount of inter-band absorption, the dielectric function is defined by the Drude and Lorentz model, which elucidates the response of damped and free electrons to an applied electromagnetic field [32]:

$$
\begin{gathered}
\varepsilon_{m}(\omega)=\varepsilon_{r}(\omega)+i \varepsilon_{i}(\omega) \\
\varepsilon_{\mathrm{m}}(\omega)=1-\frac{\omega_{\mathrm{p}}^{2}}{\omega(\omega+\mathrm{i} \gamma)}+\omega_{\mathrm{IB}}(\omega)
\end{gathered}
$$


The coupling strength between photons and the LSPR is determined by the absorption and the scattering cross-sections of the nanoparticles [33]. In equation 2.2, the Drude model for metal is used to describe the inter-band transition [34]. $\omega_{p}$ is called the plasma frequency and is defined by $\omega_{p}=\left(\frac{N_{e}{ }^{2}}{m_{e} \varepsilon_{0}}\right)^{\frac{1}{2}}$, which $\mathrm{N}_{\mathrm{e}}$ is the density of free electrons, e is the charge, $m_{e}$ is the mass of electrons, $\varepsilon_{0}$ is the dielectric constant in free space, $\gamma$ is the attenuation constant. The second term in Equation 2.2, the Lorentz, describes the inter-band transition for particles, such as holes in the occupied or unoccupied states [31]. The Electronic inter-band transitions significantly influence the surface plasmon resonance in metallic nanoparticles. As soon as the nanoparticle size is much smaller than the incident wavelength, the scattering $\left(C_{\text {scat }}\right)$ and the absorption $\left(C_{a b s}\right)$ cross-sections of spherical nanoparticles can be defined as follows:

$$
\begin{gathered}
\mathrm{C}_{\text {scat }}=\frac{1}{6 \pi}\left(\frac{2 \pi}{\lambda}\right)^{4}|\alpha|^{2} \\
\mathrm{C}_{\mathrm{abs}}=\frac{2 \pi}{\lambda} \operatorname{Im}[\alpha] \\
\alpha=4 \pi \mathrm{a}^{3}\left(\frac{\varepsilon_{\mathrm{m}}-\varepsilon_{\mathrm{d}}}{\varepsilon_{\mathrm{m}}+2 \varepsilon_{\mathrm{d}}}\right)
\end{gathered}
$$

Where $\alpha$ is the polarizability of the spherical nanoparticle with radius a, $\varepsilon_{\mathrm{m}}$ is the dielectric constant of the metal, $\varepsilon_{\mathrm{d}}$ is the dielectric constant of the environment. If resonance occurs in the system when the following condition is met and the denominator of Equation 2.5 tends to zero [34,35]. If $\varepsilon_{\mathrm{m}}=-2 \varepsilon_{\mathrm{d}}$, the polarization of the particle turns out to be considerable, and the frequency is the same as the SPR $\omega_{\text {sp }}$ permitting light to interact over a bigger region than the cross-section of the nanoparticle. The scattering efficiency can be calculated using the following formula:

$$
Q_{\text {scat }}=\frac{C_{\text {scat }}}{\left(C_{\text {scat }}+C_{a b s}\right)}
$$

Silver nanoparticles in the air have a scattering cross-section approximately ten times larger than larger particles, allowing them to fully absorb and scatter incident light with just ten percent metallic nanoparticles covering the substrate [34]. Therefore, the bigger the scattering cross-section, the more light trapping is happened by a scattering effect, and more important than the light absorption. The 
particles bigger than $100 \mathrm{~nm}$, this effect can be achieved and is suitable for the solar cell device. In addition, the shape, material of the particles, and surrounding medium contributed to enhancing the scattering effect [10,36-38]. Metal nanoparticles directly increase the absorption in the solar cells, which occurs through coupling to near fields [34]. In addition, the absorption in the near field of metal nanoparticles is about hundreds or thousands of times higher than in the surrounding field. The near field strength around nanoparticles depends on the following factors [39]: the stored energy in plasmonic modes, the sharpness and edge of the nanostructure, hence the sharper the edges in the nanostructure, the higher the near field strength around it. The stored energy also depends approximately on the cross-sectional area of the absorption on quasi-static particles (where the size of the particle is much smaller than the incident photon wavelength) [40].

Generally, metallic nanoparticles are made of silver, aluminum, gold or copper due to their strong light. SPR of aluminum and silver occurs in the ultraviolet region, while SPR of gold and copper happens in the visible region [35]. Mostly, silver nanoparticles for their low cost as well as low absorption are employed in OPV, although gold nanoparticles have recently been largely used due to not having oxidation effects.

Although the use of nanoparticles in solar cells increases the absorption of photons in the cell, mostly, a semiconductor is coated on the nanoparticles to protect them from corrosion and recombination. Semiconductors such as $\mathrm{TiO}_{2}, \mathrm{SiO}_{2}$ and $\mathrm{Al}_{2} \mathrm{O}_{3}$ coated on gold and silver nanoparticles as a protective layer are employed in the solar cells [41]. Altering the thickness of the coating layer and the dielectric of the surrounding environment $\left(\varepsilon_{d}\right)$ and the dielectric constant of the coating layer $\left(\varepsilon_{c}\right)$ are influenced by the resonance, Therefore the thickness of the coating layer increases, the resonance shifts toward the higher wavelengths. Consequently, the longer the wavelength, the greater the number of photons and the higher the intensity can be achieved. The wavelength intensity and position of the SPR depend on the dielectric function of the metal and is expressed by Equation $2.2[31,34]$.

\subsection{The Finite Difference Time Domain (FDTD) Method}

In this method, the understudied structure and interacting fields are defined by discretizing the simulation space and allocation of optical properties to cells of the 
mesh. The technique was introduced in 1966 by YEE [42] but did not receive much attention until the late 1980s owing to lack of access to computers with adequate speed and memory.

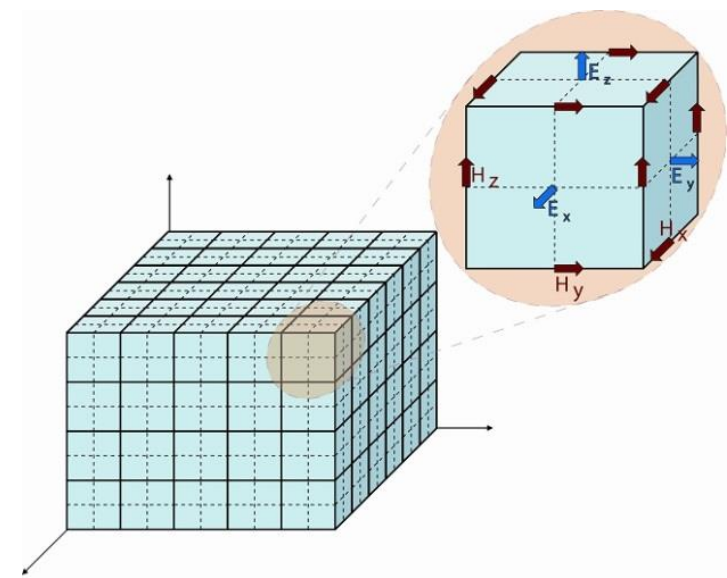

Fig. 1 Gridding in 3-dimensional FDTD space and position of components of electric and magnetic fields onto each cell

With advances in the production of personal computers, this technique came to prominence for studying propagation and interaction of electromagnetic waves and matter in many branches of science, including: physics, electronics, communications, photonics, etc. [43].

In the standard FDTD method, simulation space is divided into identical cubic cells as shown in fig. 1 Positions of components of electric and magnetic fields on each cell are alternately determined in a way that each component of the electric field is enclosed by components of the magnetic field and vice versa. By discretizing Maxwell's equations through applying finite-difference on spatial and time components of the field, six updating equations are achieved for six components of electric and magnetic fields.

The structure is discretized with a non-uniform grid mesh, which is subdivided into rectangular unit cells. The $\mathrm{H}$-and E-field field are shown in time steps, where during each time step they are iteratively updated. To avoid the reflections at the domain boundary, perfectly matched layers (PML) are applied. A three-dimensional nonuniform lattice is introduced as follows. The vertices of the lattice are defined by the one-dimensional coordinates:

$$
\left\{\mathrm{x}_{\mathrm{i}}, \mathrm{i}=1, \mathrm{~N}_{\mathrm{x}}\right\} ; \quad\left\{\mathrm{y}_{\mathrm{j}}, \mathrm{j}=1, \mathrm{~N}_{\mathrm{y}}\right\} ; \quad\left\{\mathrm{z}_{\mathrm{k}}, \mathrm{k}=1, \mathrm{~N}_{\mathrm{z}}\right\}
$$

The cell and edge centers in the non-uniform space are introduced as: 


$$
\mathrm{x}_{\mathrm{i}+1 / 2}=\mathrm{x}_{\mathrm{i}}+\Delta \mathrm{x}_{\mathrm{i}} / 2 ; \quad \mathrm{y}_{\mathrm{j}+1 / 2}=\mathrm{y}_{\mathrm{j}}+\Delta \mathrm{y}_{\mathrm{j}} / 2 ; \quad \mathrm{z}_{\mathrm{k}+1 / 2}=\mathrm{z}_{\mathrm{k}}+\Delta \mathrm{z}_{\mathrm{k}} / 2
$$

The E- and $\mathrm{H}$-field in the non-uniform grid are denoted as follow:

$$
\begin{aligned}
& \left.E_{x}\right|_{i+1 / 2, j, k} ^{n} \equiv E_{x}\left(x_{i+1 / 2}, y_{j}, z_{k}, n \Delta t\right) \\
& \left.\mathrm{H}_{\mathrm{x}}\right|_{\mathrm{i}, \mathrm{j}+1 / 2, \mathrm{k}+1 / 2} ^{\mathrm{n}+1 / 2} \equiv \mathrm{H}_{\mathrm{x}}\left[\mathrm{x}_{\mathrm{i}}, \mathrm{y}_{\mathrm{j}+1 / 2}, \mathrm{z}_{\mathrm{k}+1 / 2},(\mathrm{n}+1 / 2) \Delta \mathrm{t}\right]
\end{aligned}
$$

This algorithm is based on a discretization of Maxwell's equations in their integral form [16]. The surface integrals are performed over a lattice cell face, and contour integral is performed over the edges bounding face. The equation over cell faces using the discrete field approximations in Equation (2.9) and evaluating the time derivatives using central difference approximations leads to:

$$
\begin{aligned}
& \left.E_{x}\right|_{i+1 / 2, j+1, k} ^{n} \Delta x_{i}-\left.E_{x}\right|_{i+1 / 2, j, k} ^{n} \Delta x_{i}-\left.E_{y}\right|_{i+1, j+1 / 2, k} ^{n} \Delta y_{j}+\left.E_{y}\right|_{\left.\right|_{i, j+1 / 2, k} ^{n}} ^{n} \Delta y_{j}= \\
& -\left[\mu_{\mathrm{i}+1 / 2, \mathrm{j}+1 / 2, \mathrm{k}}\left(\frac{\left.\mathrm{H}_{\mathrm{z}}\right|_{\mathrm{i}+1 / 2, \mathrm{j}+1 / 2, \mathrm{k}} ^{\mathrm{n}+1 / 2}-\left.\mathrm{H}_{\mathrm{z}}\right|_{\mathrm{i}+1 / 2, \mathrm{j}+1 / 2, \mathrm{k}} ^{\mathrm{n}-1 / 2}}{\Delta \mathrm{t}}\right)+\left.\mathrm{M}\right|_{\mathrm{i}+1 / 2, \mathrm{j}+1 / 2, \mathrm{k}} ^{\mathrm{n}}\right] \Delta \mathrm{x}_{\mathrm{i}} \Delta \mathrm{y}_{\mathrm{j}} \\
& \left.H_{x}\right|_{i, j+1 / 2, k+1 / 2} ^{n+1 / 2} h_{x_{i}}-\left.H_{x}\right|_{i, j-1 / 2, k+1 / 2} ^{n+1 / 2} h_{x_{i}}-\left.H_{y}\right|_{i+1 / 2, j, k+1 / 2} ^{n+1 / 2} h_{y_{j}}+\left.H_{y}\right|_{i-1 / 2, j, k+1 / 2} ^{n+1 / 2} h_{y_{j}}= \\
& -\left[\begin{array}{l}
\varepsilon_{\mathrm{i}, \mathrm{j}, \mathrm{k}+1 / 2}\left(\frac{\left.\mathrm{E}_{\mathrm{z}}\right|_{\mathrm{i}, \mathrm{j}, \mathrm{k}+1 / 2} ^{\mathrm{n}+1}-\left.\mathrm{E}_{\mathrm{z}}\right|_{\mathrm{i}, \mathrm{j}, \mathrm{k}+1 / 2} ^{\mathrm{n}}}{\Delta \mathrm{t}}\right) \\
+\frac{\sigma_{\mathrm{i}, \mathrm{j}, \mathrm{k}+1 / 2}}{2}\left(\frac{\left.\mathrm{E}_{\mathrm{z}}\right|_{\mathrm{i}, \mathrm{j}, \mathrm{k}+1 / 2} ^{\mathrm{n}+1}+\left.\mathrm{E}_{\mathrm{z}}\right|_{\mathrm{i}, \mathrm{j}, \mathrm{k}+1 / 2} ^{\mathrm{n}}}{\Delta \mathrm{t}}\right)+\left.\mathrm{J}\right|_{\mathrm{i}, \mathrm{j}, \mathrm{k}+1 / 2} ^{\mathrm{n}+1 / 2}
\end{array}\right] \mathrm{h}_{\mathrm{x}_{\mathrm{i}}} \mathrm{h}_{\mathrm{y}}
\end{aligned}
$$

Where $\mathrm{h}_{\mathrm{i}}^{\mathrm{x}}=\left(\Delta \mathrm{x}_{\mathrm{i}}+\Delta \mathrm{x}_{\mathrm{i}-1}\right) / 2 ; \mathrm{i}=2, \mathrm{~N}_{\mathrm{x}}$ and the other component is introduced in the same manner. $\varepsilon_{\mathrm{i}, \mathrm{j}, \mathrm{k}+1 / 2}, \sigma_{\mathrm{i}, \mathrm{j}, \mathrm{k}+1 / 2}$ and $\mu_{\mathrm{i}+1 / 2, \mathrm{j}+1 / 2, \mathrm{k}}$ are the averaged permittivity, conductivity and permeability about grid edges, respectively. Similar updates for other components are easily derived.

Particularly for three dimensions simulation, this process is very time-consuming and requires a lot of computer memory resources. To acquire good stability between accuracy and computational resources, 10 grid points per lattice constant "a" is utilized. Several detectors are used to record the electric and magnetic fields of light. A commercial 3D-FDTD code is used, (Lumerical) to enable us the free positioning, moving, and tilting of the structure. The power flow is calculated as follows:

$$
S=\frac{\int\left(\vec{E}_{0} \times \vec{E}\right) d \vec{A}}{\int(\vec{E} \times \vec{E}) d \vec{A}}
$$

\subsection{OPV Solar Cells}


Organic Solar Cells, which are called Organic Photovoltaics (OPV), have been considerably used by industry and researchers in the recent past [44-47]. The five critical procedures for an effective OPV are summarized as 1. Light absorption goes together with exciton's creation, 2. Diffusion of the excitons through active layer interface, 3 . the dissociation and separation of charge, 4 . Transporting the separated charges, 5. Charge gathering (fig. 2) [48].

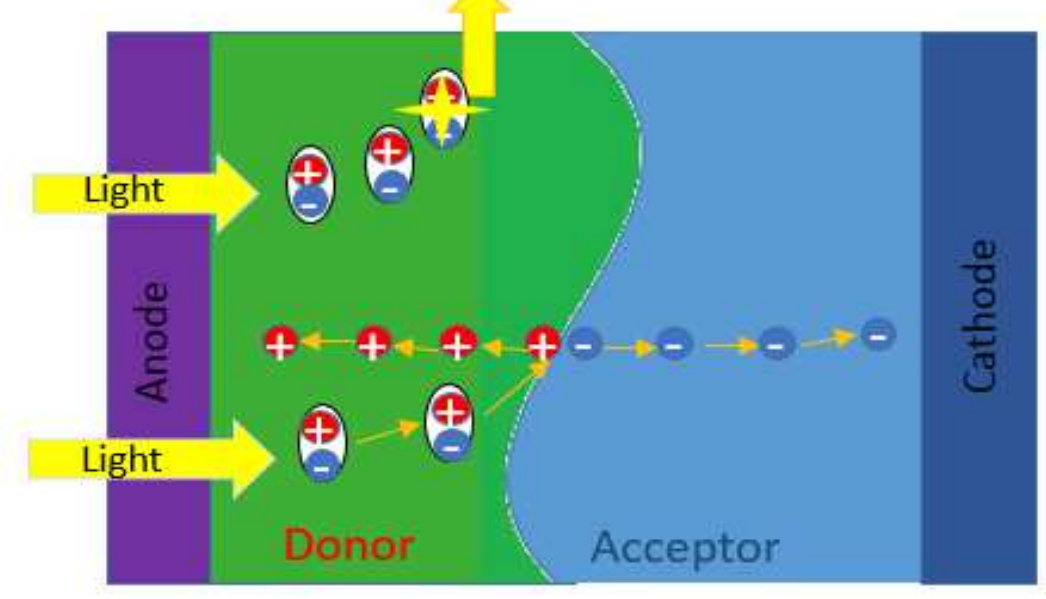

Fig. 2 Schematic of OSC principle operation

Carbon-based organic compounds, or polymers are commonly used as active semiconducting materials in organic or polymer solar cells, which can convert solar energy into electric energy. Three types of OPV are categorized as single-layered, bilayer, and bulk heterojunction structured cells. The first group of OPVs is mainly made of single organic layers inserted between two metal electrodes [49]. Since the tremendously low charge separation is caused by the presence of tightly bound excitons in organic semiconductors, these layers, as p-type or n-type sandwiches between electrodes are non-capable of appropriately creating charges in the singlelayer structure [50]. Kippelen B et al. reported single-layer OPVs with efficiencies as high as $5.9 \%$ recently [51].

In bilayer/multilayer OPVs, the active layers containing donor and, acceptor organic semiconductors absorb light and as a result, generate photocurrents. The donor material is capable of donating electrons, resultant holes, while the acceptor materials can accept electrons. Photons from light are collected by these active layers to form excitons, which produce a concentration gradient, to diffuse them towards 
the donor or acceptor interface and separate them into free holes and electrons. Therefore, free electrons and holes transfer to the electrodes, resulting in photovoltaic. In a bilayer heterojunction device, $\mathrm{p}$ and $\mathrm{n}$-type materials are consecutively placed with each other [50].

The third one, bulk heterojunction is arranged by bulk volume mixing of donor and acceptor components, which outcomes in very slight exciton diffusion length at their interface [49]. Excitons are formed as soon as photons are absorbed by organic materials. Finally, separation of excitons bounces charge gathering at the heterojunction interface at the electrodes, resulting in photovoltaic [50].

Although the inorganic semiconductor has a suitable bandgap, the main problem with them is, they possess less light absorptivity than organic materials [44]. Therefore, inorganic semiconductors require a bigger absorbing layer, producing thicker devices. Moreover, one of the vital features of inorganic semiconductors is purity in attaining higher performance, which increases the cost of production. The inorganic semiconductors, alternatively, have a lower energy bandgap for creation of excitons to produce charge carriers. As a result, for fabricating OPVs, we should attempt better electron and hole mobility, which will consequence in higher performance.

\subsection{MODEL CONFIGURATION}

Fig. 3 depicts the schematic structure of an OPV solar cell, where a hexagonal periodic array of nanoparticles is embedded inside the P3HT: PCBM active layer on the top of aluminum. The PML boundary conditions on the bottom of the aluminum layer were used to avoid reflections in the stack direction. To create multiple scattering and cross-linking between adjacent nanoparticles, the boundary conditions along the $\mathrm{X}$ and $\mathrm{Y}$ directions were considered periodically. The simulation mesh size was fixed at $0.5 \mathrm{~nm}$ in the region encircling the nanoparticles. The plane wave was illuminated at the normal incidence along the $\mathrm{z}$-direction to propagate through the structure, with a wavelength range between 300 and $900 \mathrm{~nm}$. The OPV solar cell is designed of three thin layers, which includes an aluminum substrate as a cathode with a thickness of $100 \mathrm{~nm}$, the active layer of poly trihexyl thiophene (P3HT: PCBM) with a thickness of $40 \mathrm{~nm}$, a highly conductive polymer 
layer, and the polyethylene dioxide thiophene: polysulfonate (PEDOT: PSS) as the anode with a thickness of $30 \mathrm{~nm}$ and also plasmonic metal nanospheres were used as a hexagonal lattice. The refractive index ( $\mathrm{n}$ for the real part and refractive index $k$ for the imaginary part) PEDOT: PCBM and P3HT: PCBM is taken from Ami abass [52]. To enhance the performance of OPV solar cells by improving light absorption, plasmonic nanocrystals were employed as a hexagonal lattice inside only the active P3HT: PCBM layer (as shown in Fig. 3). Intrinsic adsorption inside the active layer is calculated without silver material absorption on plasmonic nanocrystals. Furthermore, the increase in optical absorption in multilayer cells can be evaluated to high efficiencies.

For this calculation, the dissipated power $\mathrm{L}(\lambda)$ into the volume $(\mathrm{x}, \mathrm{y}, \mathrm{z})$ of material from the Poynting vector $\Pi(\mathrm{x}, \mathrm{y}, \mathrm{z}, \lambda)$ is expressed as following [16]:

$$
\mathrm{L}(\lambda)=\int \mathrm{x} \int \mathrm{y} \int \mathrm{z}-\operatorname{div}\{(\Pi(\mathrm{x}, \mathrm{y}, \mathrm{z}, \lambda))\} d x d y d z
$$

The intrinsic absorption $A(\lambda)$ in each layer is governed by the ratio of the $L(\lambda)$ in each volume to the total power of the incident light source $\mathrm{P}$ Source $(\lambda)$ as following (2.14) [53]:

$$
\mathrm{A}(\lambda)=\frac{\mathrm{L}(\lambda)}{\operatorname{source}(\lambda) \mathrm{P}}
$$

In the wavelength range of 300 to $900 \mathrm{~nm}$, the optical absorption enhancement $A_{E}$ is calculated as follows (2.15) [54]:

$$
\mathrm{A}_{\mathrm{E}}=\frac{\int_{\lambda 2}^{\lambda 1}\left(\left(\frac{\lambda}{h c}\right) \mathrm{A}(\lambda) \mathrm{I}(\lambda) \mathrm{d} \lambda\right)}{\int_{\lambda 2}^{\lambda 1}\left(\left(\frac{\lambda}{h c}\right) \text { Awithout } \mathrm{NSs}(\lambda) \mathrm{I}(\lambda) \mathrm{d} \lambda\right)}
$$

Where, his Planck's constant and $\mathrm{c}$ is the speed of light in vacuum, while $\mathrm{I}(\lambda)$ denotes the $\mathrm{AM}_{1.5} \mathrm{G}$ solar spectral irradiance. $\mathrm{A}(\lambda)$ and $\mathrm{A}_{\text {without NSs }(\lambda)}$ are the intrinsic optical absorption (inside the active layer) with and without nanospheres, respectively. The dissipated power ( $\mathrm{D} p$ ) via the active layer of the plasmonic nanocrystal of intrinsic absorption is defined by the $\mathrm{AM}_{1.5} \mathrm{G}$ illumination of light in the wavelength range of 300 to $900 \mathrm{~nm}[55]$.

$$
\mathrm{D}_{\mathrm{p}}=\int_{\lambda 2}^{\lambda 1} \mathrm{~A}(\lambda) \operatorname{Id} \lambda
$$

The square of the electric field integration over the volume is employed for the calculation of the absorption of the total energy of light by the substrate.

$$
\mathrm{Q}_{\text {with(without) }}(\omega)=\frac{\omega}{4 \pi} \int_{\mathrm{V}} \mathrm{n}_{3} \varepsilon_{\text {with(without) }}^{\prime \prime}(\omega) \mathrm{E}^{2}
$$


This denotes the imaginary part of the dielectric function of the substrate with and without metallic nanoparticles. The ratio of light absorbed with and without metallic nanoparticles is defined as the light absorption (efficiency).

$$
A(\omega)=\frac{Q_{\text {with }}(\omega)}{Q_{0}(\omega)}
$$

Finally, there is the absorption enhancement. For the measurement of the induced short circuit current $\mathrm{I}_{\mathrm{SC}}$ in the cell (the presence of metallic nanoparticles), an integration over the solar spectrum is used:

$$
\mathrm{I}_{\mathrm{SC}}=\frac{\int \mathrm{Q}_{\mathrm{with}}(\omega) \mathrm{F}(\omega) \mathrm{d} \omega}{\int \mathrm{Q}_{0}(\omega) \mathrm{F}(\omega) \mathrm{d} \omega^{\prime}}
$$

where $F(\boldsymbol{\omega})$ represents the AM1.5 G solar light.[56]
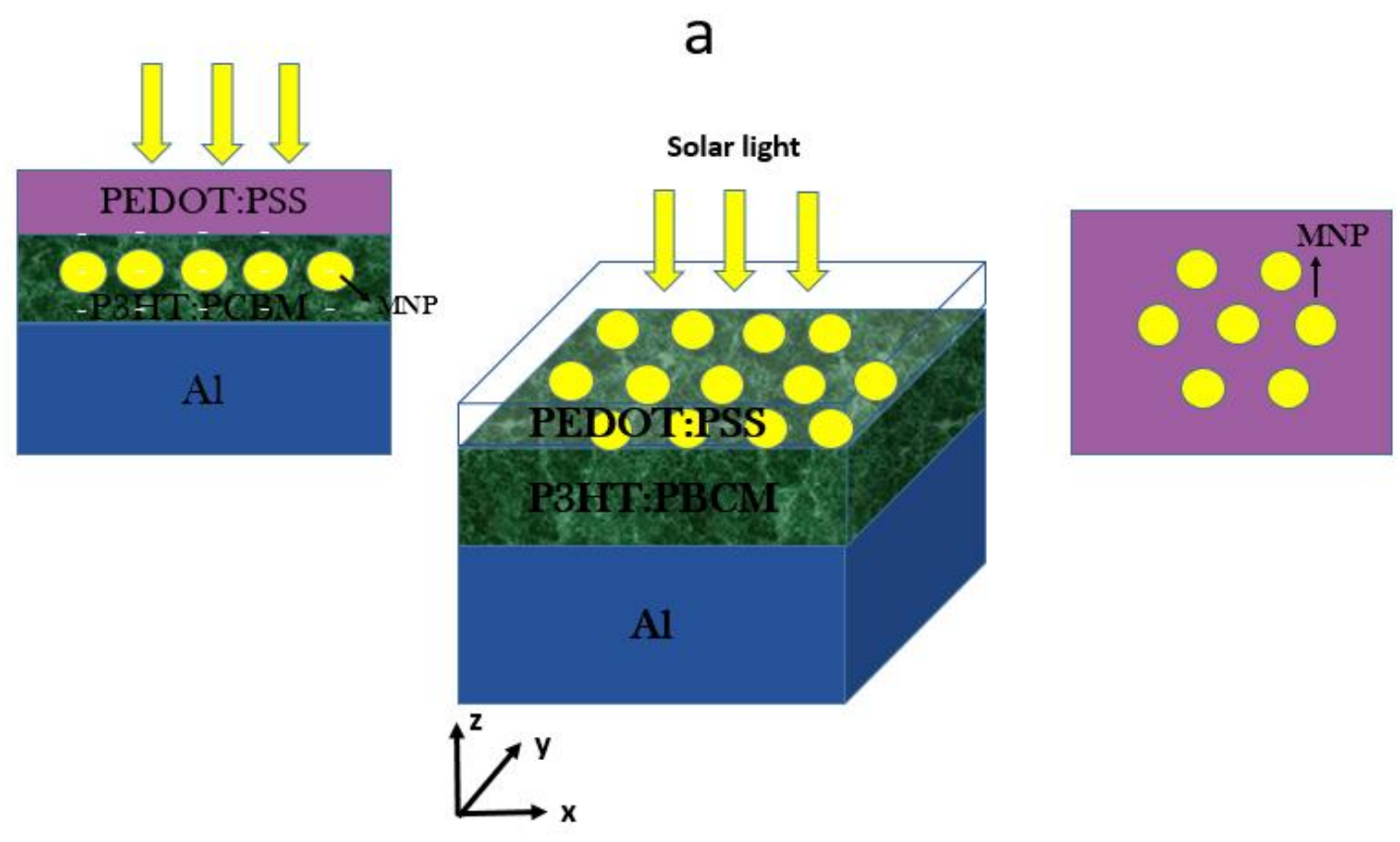


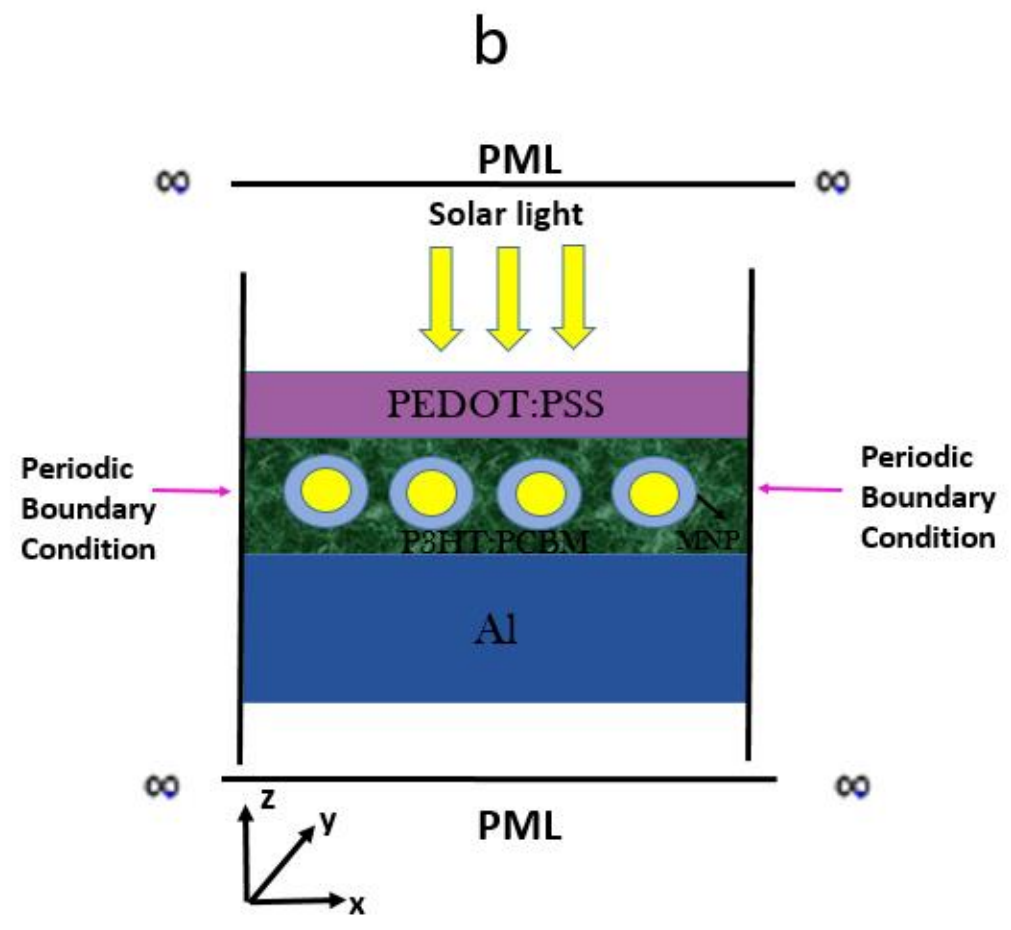

Fig. 3 a shows the structure of the reference cell. the structure of a polymer solar cell with plasmonic nanocrystal in a hexagonal lattice in three dimensions. $b$ Schematic of the proposed multilayer structure and simulation layout. The boundary condition is shown.

\section{RESULTS AND DISCUSSIONS}

The metallic nanoparticles embedded on the OPV solar cell in three dimensions are simulated using Lurmical FDTD and their plasmonic effect analyzed as the model configuration is shown in Fig. $3 \mathrm{a}$ and b. The period, size, and material of $\mathrm{Ag}$ nanoparticles are varied to study how these nanoparticles could enhance the light absorption of the OPV solar cells. The results of the simulation are illustrated in Fig. $4,5,6,7,8,9,10$. The photon absorption spectrum of OPV solar cells is plotted with and without Ag nanoparticles located inside the P3HT: PCBM in terms of wavelength at normal incidence. The simulation makes it possible to optimize the amount of light scattered into the P3HT: PCBM for several materials, periods and sizes of the metallic nanoparticles. Several parameters such as: material, alternating period and size of Ag nanoparticles, the thickness of polymer active layer, as well as dielectric shell with different thicknesses around Ag nanoparticles were investigated in detail. The wavelength range from $300 \mathrm{~nm}$ to $900 \mathrm{~nm}$ is taken into account here. 


\subsection{Ag nanoparticles embedded inside the P3HT: PCBM layer}

The comparison of light absorption of OPV solar cell with and without Ag nanoparticles revealed that the Ag nanoparticles in a hexagonal array embedded inside the P3HT: PCBM layer increased the plasmonic effect on the light absorption of OPV solar cell. Recent papers have shown that the LSP mode, which traps induces light, enhances the light absorption with plasmonic metallic nanoparticles, and their electric field inside the metallic nanoparticles is proportional to that outside [17,57]. Hence, the light absorbed inside the Ag nanoparticles, traps most of the incident light and is absorbed by the active layer of the OPV solar cellThe peak of increase in absorption due to plasmonic nanosphere crystals is observed at approximately 680 $\mathrm{nm}$. Moreover, an additional spectral peak is observed at $620 \mathrm{~nm}$. These peaks result from the LSPR between neighbouring Ag nanoparticles hexagonal array, as is shown in Fig. 3. Fig. 7 demonstrates the thickness dependence of the absorptance spectra. The increase in light absorption is due to the increase of the near field in the active P3HT: PCBM layer. The magnetic field distribution is shown in Fig. 4, The enhanced field between the plasmonic nanospheres is polarized in the direction of light.

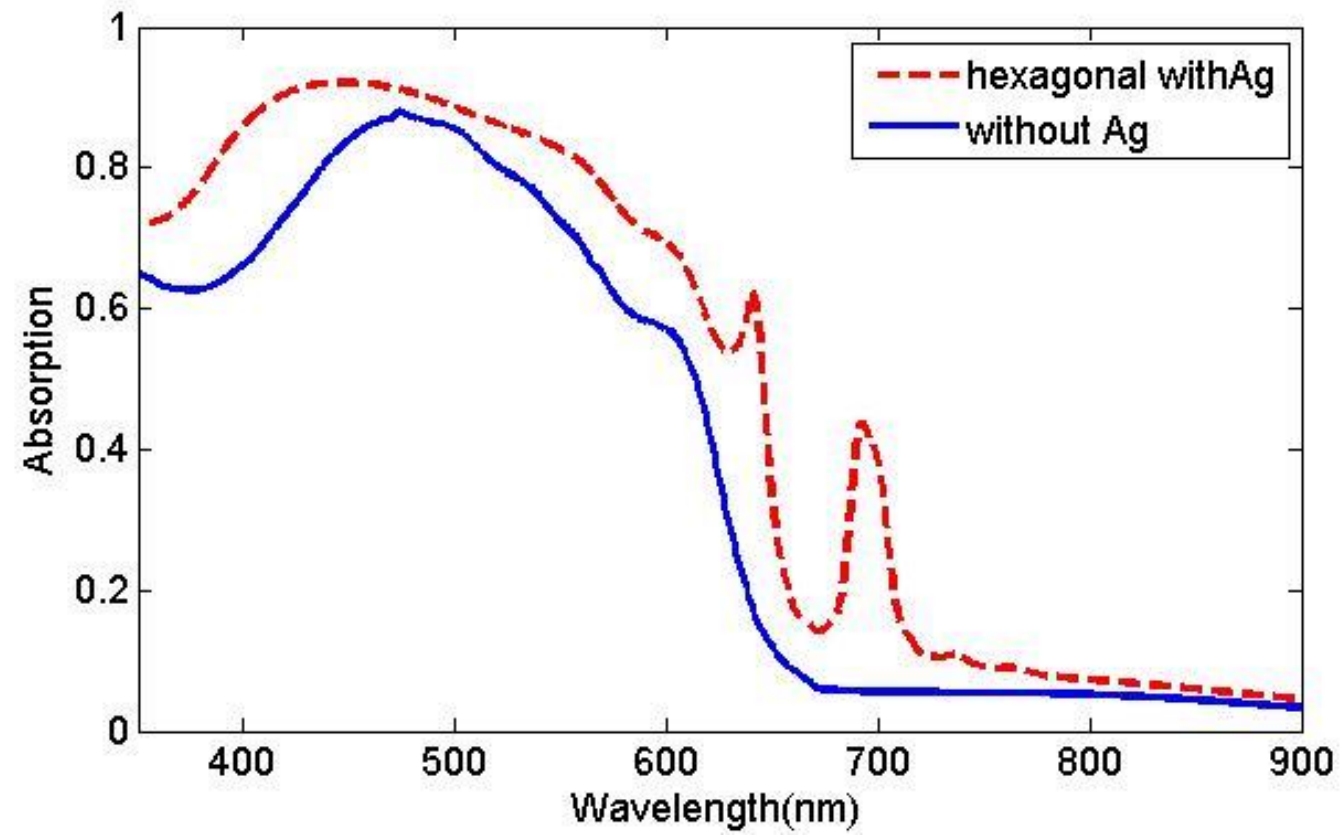

Fig. 4 The absorption spectrum of OPV solar cell with and without Ag nanoparticles located inside the P3HT: PCBM. 


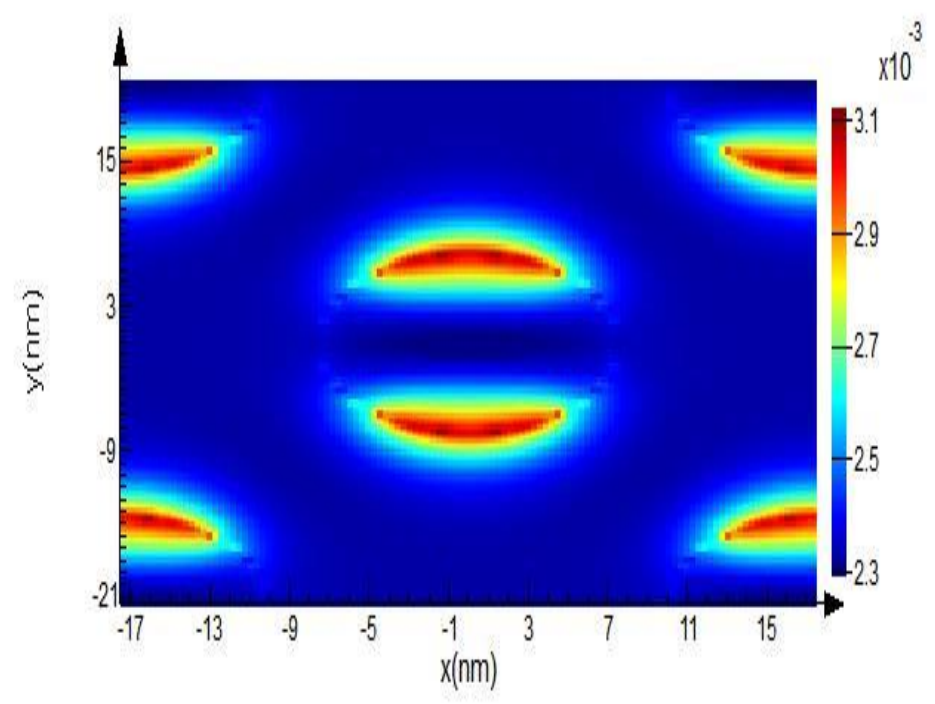

Fig. 5 The field profile of exciting mode defined for OPV solar cell with Ag nanoparticles located inside the P3HT: PCBM.

\subsection{Effect of Period on Absorption Enhancement}

The OPV solar cells were simulated with periodic arrays of Ag nanoparticles (with a radius of $15 \mathrm{~nm}$ and 25-55 nm period) embedded inside the P3HT: PCBM layer active. The effect of plasmonic enhancement by altering the period of absorption is displayed in Fig. 6. By tuning the period and size of the Ag plasmonic nanocrystals, the SPR peak can be designed to optimize the absorption of the whole incident light in the active polymer layers. Fig. 6 shows the absorption on the polymer active layer as a function of the Ag plasmonic nanocrystals period. This parameter displays the most effects on near field enhancement in Ag plasmonic nanocrystals. When the period of the nanoparticles array is much larger than the size of the nanoparticles, the absorption factor decreases, since the field scattering of the adjacent nanoparticles in the further distance is weaker. As the period of the nanoparticle array reduced, the absorption factor enhanced, as long as it reached a maximum value in a suitable period. This maximum value occurs where the field scattering is very large, where the nanoparticles are coupled to the electromagnetic field. [22,58]. As it is clear in fig 6, the optimal period of Ag plasmonic nanocrystals was observed 
around 25 to $30 \mathrm{~nm}$.

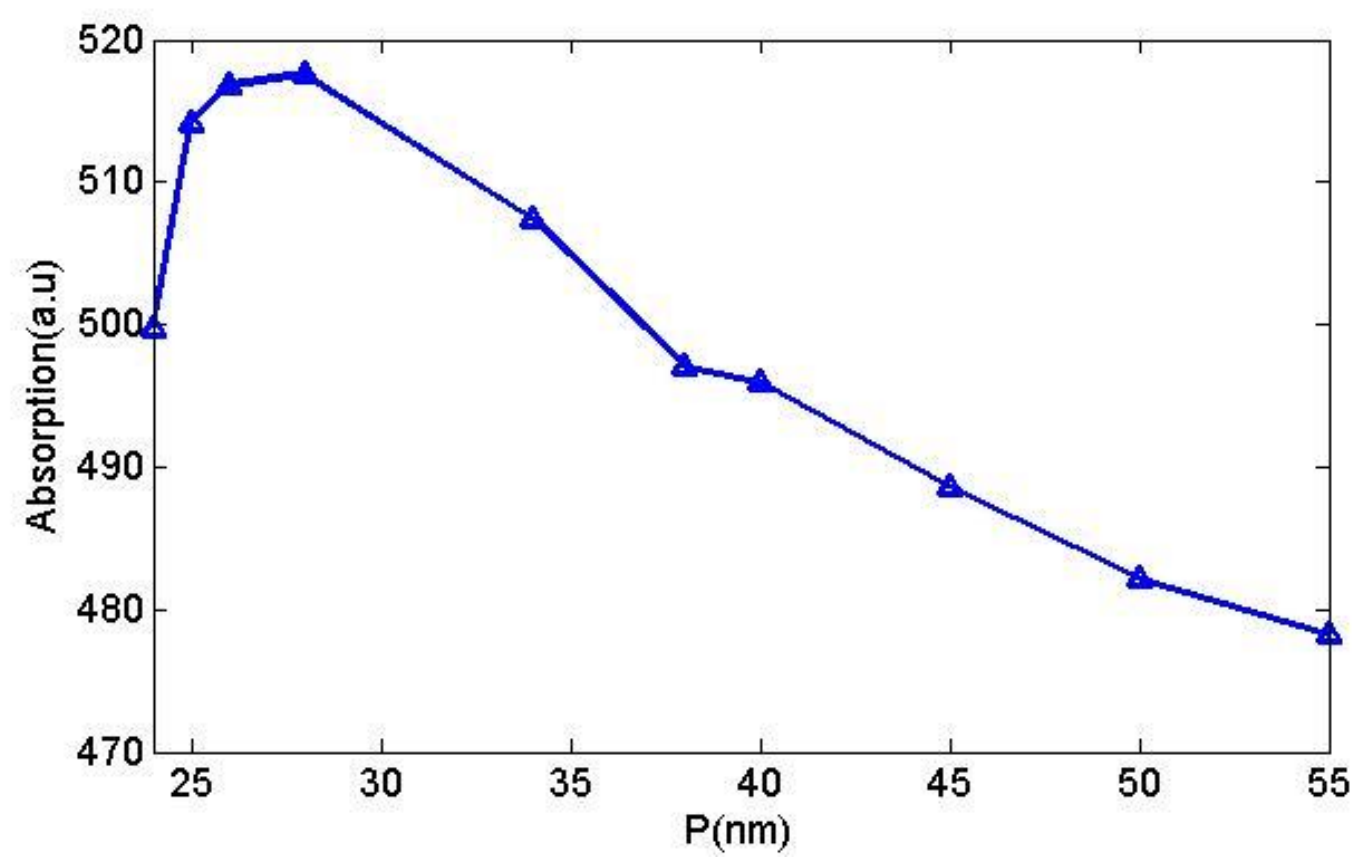

Fig. 6 Absorption of the Ag plasmonic nanocrystals with the diameter of $15 \mathrm{~nm}$ embedded inside the $40 \mathrm{~nm}$ thick P3HT: PCBM active layer in terms of different alternating periods.

\subsection{Effect of Plasmonic Nanocrystals Size}

The radius of the plasmonic nanocrystal is another significant parameter. As the radius of the Ag plasmonic nanocrystal increased, the absorption peak enhanced and shifted to higher wavelengths, as is shown in Fig. 7. It can be concluded that with growing the size of the plasmonic nanocrystal, the absorption, as well as the scattering cross-section increased. 


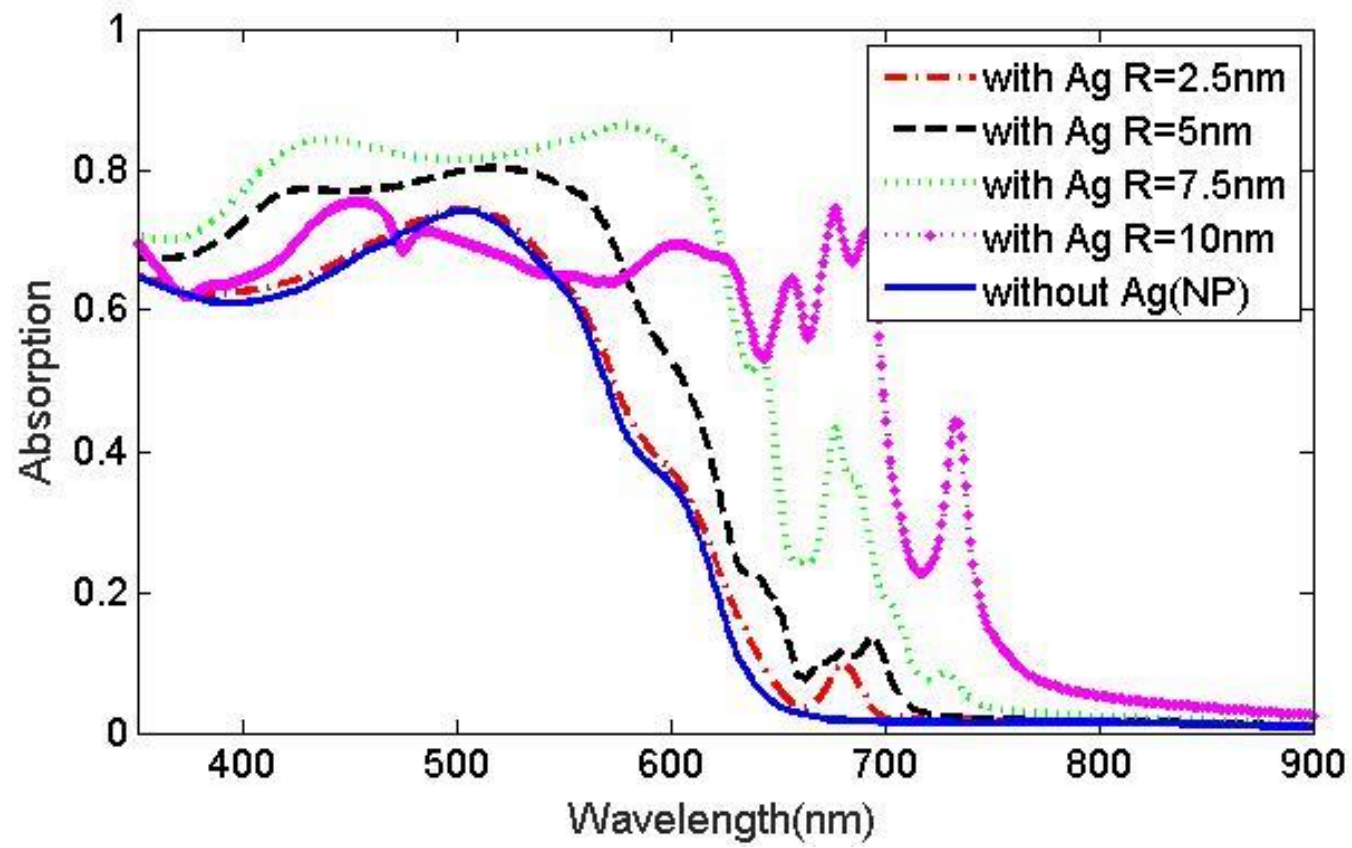

Fig. 7 Absorption of the Ag plasmonic nanocrystals with the diameters of 5, 10, 15, $20 \mathrm{~nm}$ and a period of $25 \mathrm{~nm}$ embedded inside the $40 \mathrm{~nm}$ thick P3HT: PCBM active layer in terms of wavelength.

\subsection{Effect Material Plasmonic Nanocrystals}

The absorption factor in terms of wavelength for different plasmonic nanocrystals is shown in Fig. 8. Hexagonal array of plasmonic nanocrystals with different materials such as silver, gold, aluminum, and copper embedded inside the P3HT: PCBM active layer are investigated. The results revealed that silver is the best material with higher absorption [59]. An interface Schottky barrier is formed between Ag, Au and others, since the nanoparticles are adjacent to each other. Its depth depends on the metal sizes, work functions, and accordingly, the induced electron transfer, as well as the efficiency would be higher. The separation of excited charges in the presence of an electric field improved the device's efficiency for the solar energy application. The work function 5.1 4.26, 4.67, and $4.18 \mathrm{e} \mathrm{v}$ for $\mathrm{Au}, \mathrm{Ag}, \mathrm{Cu}$, and $\mathrm{Al}$, respectively. As a result, $\mathrm{Au}$ with higher work function is more active, while their morphology, the incident light intensity, as well as excitation wavelength influence electron transfer. As long as the excitation is shifted to a higher wavelength, the steady-state polarization is increased, while the Fermi levels are shifted to reach the conduction 
band of their embedded medium to enhance charge transport. The size of the metallic nanoparticles is shifted the Fermi level to upward or downward. Since nanoparticles are well attached to the surface of the embedded medium in comparison to the large particles, thus, the Fermi level is shifted from more negative level, which could be tuned to a appropriate location by choosing and controlling the actual material, shape, as well as, size of the metallic nanoparticles.

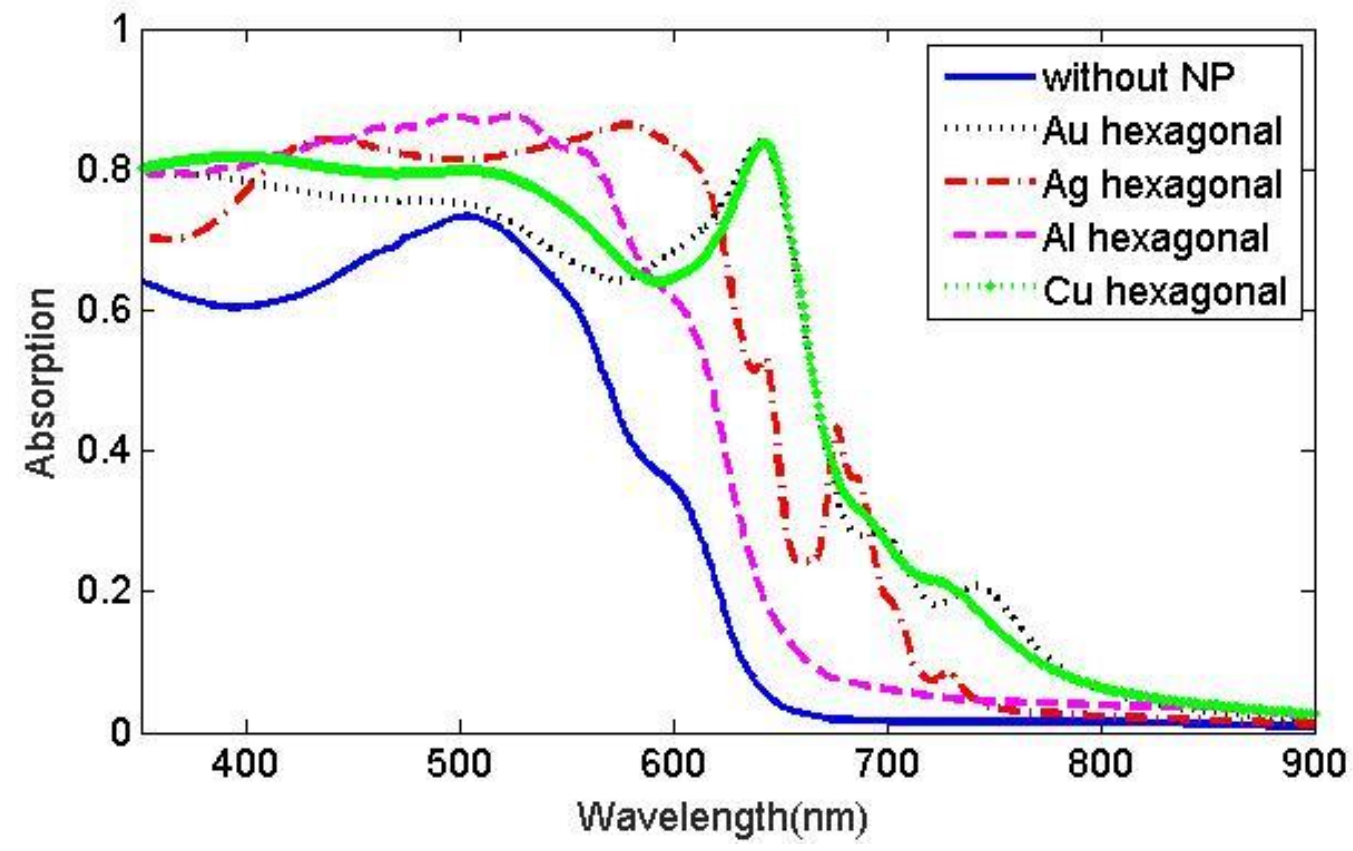

Fig. 8 Absorption of the Ag plasmonic nanocrystals with the diameter of $15 \mathrm{~nm}$ and period $25 \mathrm{~nm}$ embedded inside the $40 \mathrm{~nm}$ thick P3HT: PCBM active layer in terms of wavelength.

\subsection{Effect of the Polymer active layer Thickness on Absorption Enhancement}

The absorption plasmonic nanocrystals in the P3HT: PCBM active layer is simulated as a function of the thickness of the active layer, as shown in Fig. 9. The P3HT: PCBM layer usually makes better hole transport from the active layer to the anode, This active layer is presented in the OPV solar cell with a thickness of $40 \mathrm{~nm}$. The thickness variation of the P3HT: PCBM active layer from 20 to $70 \mathrm{~nm}$ is chosen, which has not observed substantial improvement from 40 to $70 \mathrm{~nm}$ on the absorption 
in the P3HT: PCBM active layer, as shown in Fig. 9. However, as the thickness of the P3HT: PCBM active layer increases from $20 \mathrm{~nm}$ to $70 \mathrm{~nm}$, the amount of absorption increases. By the growing thickness of the active layer, the multiple peaks correspond to the LSPR inside or between neighbouring holes. In addition, the resonances get weaker as thickness grows due to less light-matter interaction. Therefore, we expect that the sensitivity to the thickness variation in the optimum active layer is small.

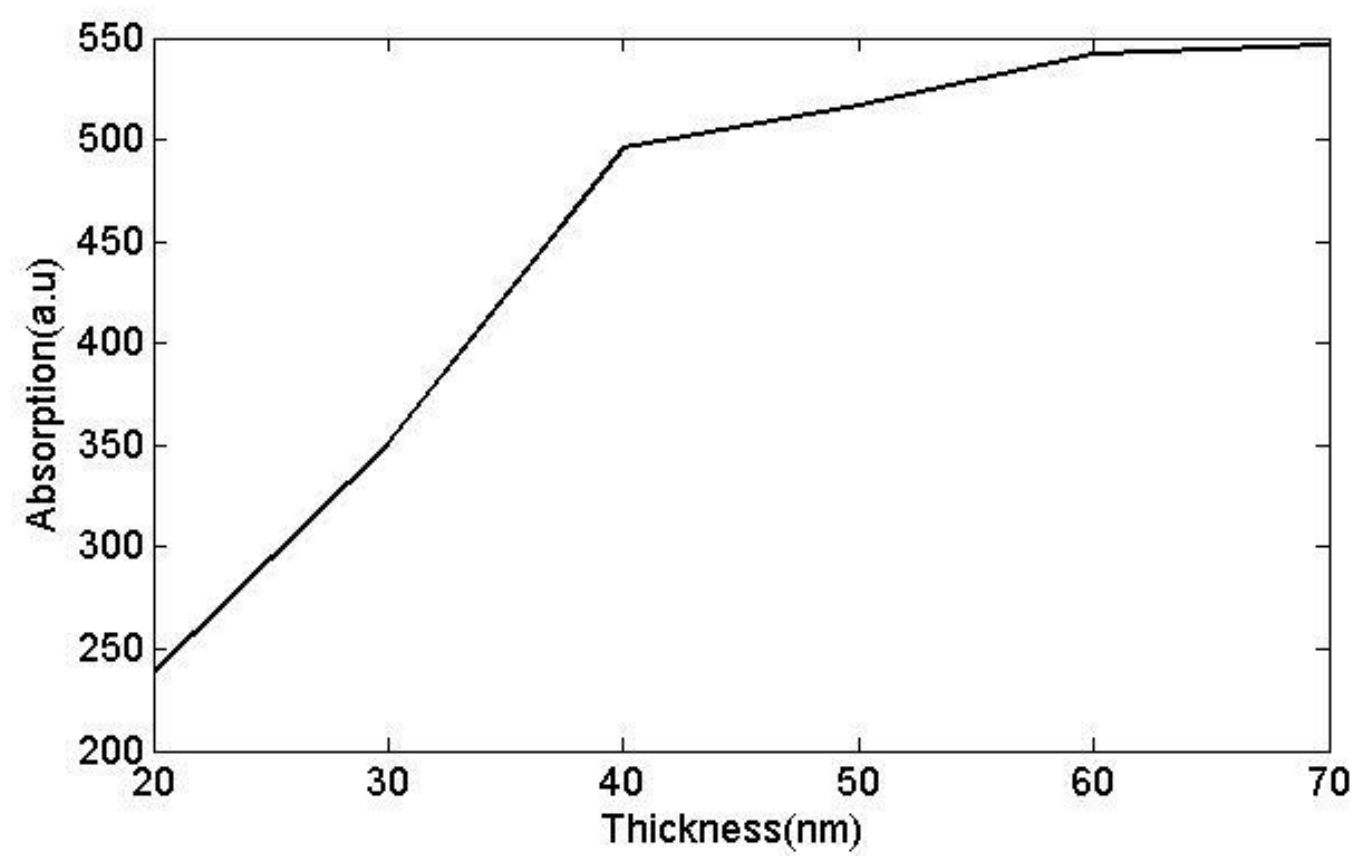

Fig. 9 shows the light absorption of an OPV solar cell in terms of the thickness of the polymer active layer.

\subsection{Effect of The Hexagonal plasmonic nanocrystals Shell Thickness on Absorption Enhancement}

Finally, the increase of light absorption is investigated by using plasmonic nanocrystals of silver nanospheres with dielectric shells. In some electron-hole recombination materials from plasmonic nanocrystals in application systems, they may significantly reduce conversion efficiency by LSPR, another reason for the use of nanoshells in applications to prevent plasmonic nanoparticles corrosion. To solve this problem, nanospheres are separated from the active polymer layer by dielectric shells. 
Dielectric shells with different refractive indices and thicknesses also change the location of the SPR peak [60]. Figure 8 shows the absorption factor in the P3HT: PCBM active layer as a function of shell thickness using two dielectric shells made of $\mathrm{SiO}_{2}$, and $\mathrm{Al}_{2} \mathrm{O}_{3}$. The absorption factor decreases with increasing thickness for both shells. This decrease is more severe for $\mathrm{SiO}_{2}$ than for $\mathrm{Al}_{2} \mathrm{O}_{3}$. This is because the change in shorter wavelengths for silicon dioxide is larger than for $\mathrm{Al}_{2} \mathrm{O}_{3}$, and this is because $\mathrm{SiO}_{2}$ has a smaller refractive index.

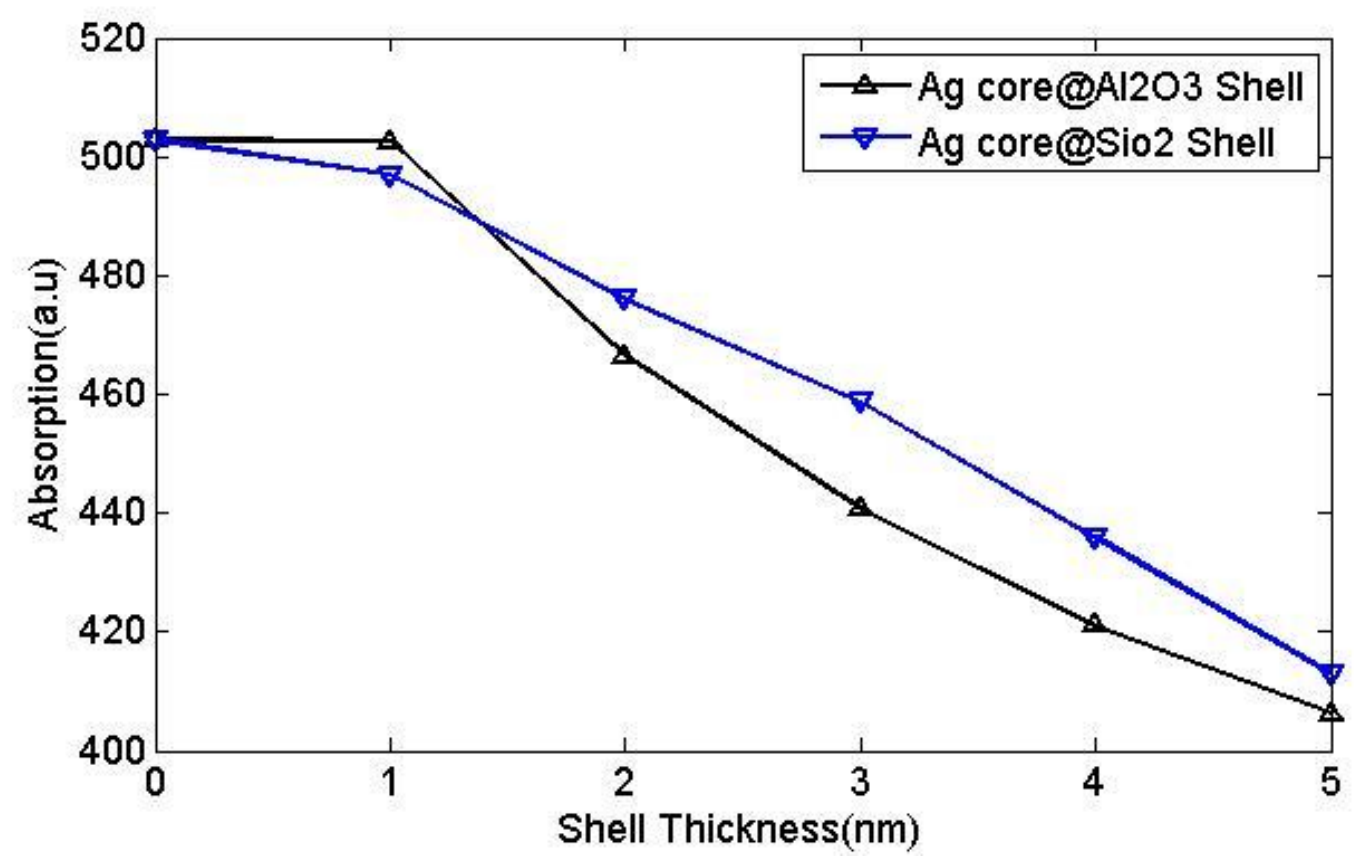

Fig.10 Absorption of the Ag plasmonic nanocrystals with the diameter of $15 \mathrm{~nm}$ and period 35 $\mathrm{nm}$ embedded inside the $40 \mathrm{~nm}$ thick P3HT: PCBM active layer in terms of dielectric shell thickness of $\mathrm{SiO}_{2}$, and $\mathrm{Al}_{2} \mathrm{O}_{3}$.

As a result, the surface plasmon resonance improves the enhancement of the scattering and absorption cross-sections of the metallic nanoparticles. Meanwhile, scattering and absorption procedures are happened, through radiative and nonradiative plasmon decay processes, respectively, their decays rate ratio depends on the nanoparticles morphology nanoparticles. The radiative plasmon decay originates from the scattering, and it is more usual in Ag nanoparticles with larger sizes. The nonradiative plasmon decay originates from the absorption, which leads to the robust absorption of light and happens via intra-band excitations within the conduction band or inter-band excitations below the conduction band. It is more 
common in smaller sizes of Ag nanoparticles. The robust absorption of light leads to more electron-hole pairs production, consequently, enhances the light absorption, as well as efficiency of the OPV solar cell.

\subsection{Photovoltaic performance}

As discussed later, absorption enhancement is mostly attained in the short wavelength range using hexagonal Ag plasmonic nanocrystals embedded inside the P3HT: PCBM active layer in fig 2. Although Ag plasmonic nanocrystals influence the short wavelength region, the main enhancement occurs at long wavelengths. OPV solar cell fabrication using Ag plasmonic nanocrystals as an absorber in the active layer is analyzed by using the advantage of the density of state of $\mathrm{Ag}$ plasmonic nanocrystals by arranging their bandgap in the non-absorbing region at the weakly absorbing region. Table 1 summarized the characteristics of the I-V curve of the OPV solar cell structure with and without Ag plasmonic nanocrystals. It shows a clear improvement in $\mathrm{J}_{\mathrm{SC}}$ of the OPV solar cells in comparison to the cell without Ag plasmonic nanocrystals as a reference cell. Moreover, the characteristics of the I-V curve of all the proposed OPV solar Cells are calculated and the results are presented in Table 1. The results revealed that all physical parameters influence on absorption enhancement, Jsc and consequently, the efficiency of OPV solar cells.

Table 1: Summary of the OPV solar cell's performance.

\begin{tabular}{|c|c|c|}
\hline $\begin{array}{c}\text { Proposed OPV Structure } \\
\text { With P3HT: PCBM active layer }\end{array}$ & $\begin{array}{c}\mathrm{J}_{\mathrm{SC}} \\
\left(\mathrm{mA} / \mathrm{cm}^{2}\right)\end{array}$ & $\begin{array}{c}\text { Absorption Enhancement } \\
(\%)\end{array}$ \\
\hline Without Ag plasmonic nanocrystals (Reference) & 19.7 & --- \\
\hline With Ag plasmonic nanocrystals & 24.3 & 26.6 \\
\hline Core $\mathrm{Ag}$, dielectric shell $\mathrm{SiO}_{2}$, & 25.12 & 26.1 \\
\hline Core $\mathrm{Ag}$, dielectric shell $\mathrm{Al}_{2} \mathrm{O}_{3}$ & 25.01 & 26.0 \\
\hline thickness of the polymer active layer 20 & 11.6 & -34.2 \\
\hline thickness of the polymer active layer 50 & 16.9 & -14.6 \\
\hline thickness of the polymer active layer 70 & 24.0 & 26.3 \\
\hline thickness of the polymer active layer 80 & 26.7 & 32.7 \\
\hline materials: silver nanoparticles & 24.3 & 26.6 \\
\hline
\end{tabular}




\begin{tabular}{|c|c|c|}
\hline materials: gold nanoparticles & 24.6 & 26.9 \\
\hline materials: aluminum nanoparticles & 24.7 & 27 \\
\hline materials: copper nanoparticles & 24.2 & 26.4 \\
\hline radius of Ag nanoparticles $2.5 \mathrm{~nm}$ & 19.8 & 3 \\
\hline radius of Ag nanoparticles $5 \mathrm{~nm}$ & 21.3 & 15.4 \\
\hline radius of Ag nanoparticles $7.5 \mathrm{~nm}$ & 23.1 & 19.5 \\
\hline radius of Ag nanoparticles $10 \mathrm{~nm}$ & 24.4 & 26.8 \\
\hline Period of Ag plasmonic nanocrystals $25 \mathrm{~nm}$ & 23.5 & 24.8 \\
\hline Period of Ag plasmonic nanocrystals $30 \mathrm{~nm}$ & 26 & 31.4 \\
\hline Period of Ag plasmonic nanocrystals $35 \mathrm{~nm}$ & 23.7 & 21.6 \\
\hline Period of Ag plasmonic nanocrystals $45 \mathrm{~nm}$ & 20.5 & 12.1 \\
\hline
\end{tabular}

\section{Conclusion}

Light absorption enhancement by an Ag plasmonic nanocrystals hexagonal array embedded inside the P3HT: PCBM active layer at the bottom of the OPV solar cell was analyzed. FDTD simulations were performed to further investigate the influence of different geometry parameters on the absorption enhancement. Simulation results revealed that $\mathrm{Ag}$ plasmonic nanocrystals can significantly improve total absorption by exciting numerous optical resonances. The outcome of each active layer and nanoparticle morphology design on the absorption enhancement was debated. Light scattering by Ag plasmonic nanocrystals enhances light absorption in the long wavelength range, and also generates a strong electromagnetic field.

As proved in the simulation, plasmonic nanostructures with various morphology parameters such as size, materials, display different behavior on absorption enhancement. For instance, a different range of $\mathrm{Ag}$ nanoparticles embedded in the P3HT: PCBM active layer, using Lumerical FDTD, makes it possible to estimate the amount of light scattered into the P3HT: PCBM active layer for various materials and sizes of the Ag nanoparticles. It was seen that the scattered light scattered inside the active layer was influenced by the size, the material, and embedded environment of the Ag nanoparticle. Finally, the resonant plasmon excitation in an OPV solar cell is to exploit the strong local field enhancement around the Ag nanoparticles, which improves the light absorption in the P3HT: PCBM active layer. In conclusion, we have revealed that the $\mathrm{I}_{\mathrm{SC}}$, consequently, efficiency, was enhanced due to light scattering, strong absorption and the resonant plasmon excitation of Ag plasmonic 
nanocrystals. The clear enhancement in the I-V curve is attained with the $\mathrm{Ag}$ plasmonic nanocrystals embedded in the active layer of the OPV solar cells.

\section{Conflict of Interest}

The authors declare that they have no conflict of interest.

\section{Ethical approval}

This paper does not contain any studies with human participants or animals performed by any of the authors.

\section{Funding}

Not applicable

\section{Consent to participate}

Not applicable

\section{Consent for publication}

Not applicable

\section{Authors' contributions}

A. Mohammadi designed and directed the project, and with L. Shabani, contributed to the design and implementation of the research, A. Mohammadi, L. Shabani, T. Jalali contributed to the analysis of the results and to the writing of the manuscript.

\section{Acknowledgments}

We are thankful to the Persian Gulf University Research Council for continuous support.

\section{Data and code availability}

The data that support the findings of this study are available from the corresponding author upon reasonable request.

\section{References}

[1] Gomard, Guillaume, et al. "Two-dimensional photonic crystal for absorption enhancement in hydrogenated amorphous silicon thin film solar cells." Journal of Applied Physics 108.12 (2010): 123102. https://doi.org/10.1063/1.3506702

[2] Dühring, Maria B., and Ole Sigmund. "Optimization of extraordinary optical absorption in plasmonic and dielectric structures." JOSA B 30.5 (2013): 1154-1160. https://doi.org/10.1364/JOSAB.30.001154 
[3] Han, Seok Jun, et al. "Symmetry-breaking nanostructures on crystalline silicon for enhanced light trapping in thin film solar cells." Optics express 24.26 (2016): A1586-A1596. https://doi.org/10.1364/OE.24.0A1586

[4] Bermel, Peter, et al. "Improving thin-film crystalline silicon solar cell efficiencies with photonic crystals." Optics express 15.25 (2007): 16986-17000. https://doi.org/10.1364/OE.15.016986

[5] Furumi, Seiichi. "Self-assembled organic and polymer photonic crystals for laser applications." Polymer journal 45.6 (2013): 579-593. https://doi.org/10.1038/pj.2012.181

[6] Fu, Yulan, and Tianrui Zhai. "Distributed feedback organic lasing in photonic crystals." Frontiers of Optoelectronics 13.1 (2020): 18-34.https://doi.org/10.1007/s12200-019-0942-1

[7] Yablonovitch, Eli, and George D. Cody. "Intensity enhancement in textured optical sheets for solar cells." IEEE Transactions on electron devices 29.2 (1982): 300-305. https://doi.org/10.1109/TED.1982.20700

[8] Park, Yeonsang, et al. "Absorption enhancement using photonic crystals for silicon thin film solar cells." Optics Express 17.16 (2009): 14312-143. https://doi.org/10.1364/OE.17.014312

[9] Ferry, Vivian E., et al. "Light trapping in ultrathin plasmonic solar cells." Optics express 18.102 (2010): A237-A245. https://doi.org/10.1364/OE.18.00A237

[10] Munday, Jeremy N., and Harry A. Atwater. "Large integrated absorption enhancement in plasmonic solar cells by combining metallic gratings and antireflection coatings." Nano letters 11.6 (2011): 21952201. https://doi.org/10.1021/nl101875t

[11] Nagel, James R., and Michael A. Scarpulla. "Enhanced absorption in optically thin solar cells by scattering from embedded dielectric nanoparticles." Optics express 18.102 (2010): A139-A146. https://doi.org/10.1364/OE.18.00A139

[12] Zhang, Wei, et al. "Combined front diffraction and back blazed gratings to enhance broad band light harvesting in thin film solar cells." Optics communications 298 (2013): 250-253. https://doi.org/10.1016/j.optcom.2013.02.045

[13] Krauss, Thomas F., M. Richard, and Stuart Brand. "Two-dimensional photonic-bandgap structures operating at near-infrared wavelengths." Nature 383.6602 (1996): 699-702. https://doi.org/10.1038/383699a0 
[14] Das, Sonali, et al. "Enhanced optical absorption and electrical performance of silicon solar cells due to embedding of dielectric nanoparticles and voids in the active absorber region." Journal of Modern Optics 60.7 (2013): 556-568. https://doi.org/10.1080/09500340.2013.796015

[15] Jalali, T., et al. "Efficient effective permittivity treatment for the 2D-FDTD simulation of photonic crystals." Journal of Computational and Theoretical Nanoscience 4.3 (2007): 644-648. https://doi.org/10.1166/jctn.2007.029

[16] Maier, Stefan Alexander. Plasmonics: fundamentals and applications. Springer Science \& Business Media, 2007. https://doi.org/10.1007/0-387-37825-1_2

[17] Wang, Shu-Yi, Diana-Andra Borca-Tasciuc, and Deborah A. Kaminski. "Spectral coupling of fluorescent solar concentrators to plasmonic solar cells." Journal of Applied Physics 109.7 (2011): 074910. https://doi.org/10.1063/1.3567928

[18] Kelly, K. Lance, et al. "The optical properties of metal nanoparticles: the influence of size, shape, and dielectric environment." (2003): 668-677. https://doi.org/10.1021/jp026731y

[19] Ringe, Emilie, et al. "Unraveling the effects of size, composition, and substrate on the localized surface plasmon resonance frequencies of gold and silver nanocubes: a systematic single-particle approach." The Journal of Physical Chemistry C114.29 (2010): 12511-12516. https://doi.org/10.1021/jp104366r

[20] Wang, Wenhui, and Limin Qi. "Light management with patterned micro-and nanostructure arrays for photocatalysis, photovoltaics, and optoelectronic and optical devices." Advanced Functional Materials 29.25 (2019): 1807275. https://doi.org/10.1002/adfm.201807275

[21] Zhu, Jinfeng, et al. "Plasmonic effects for light concentration in organic photovoltaic thin films induced by hexagonal periodic metallic nanospheres." Applied Physics Letters 98.15 (2011): 151110. https://doi.org/10.1063/1.3577611

[22] N'konou, Kekeli, Leo Peres, and Philippe Torchio. "Optical absorption modeling of plasmonic organic solar cells embedding silica-coated silver nanospheres." Plasmonics 13.1 (2018): 297-303. https://doi.org/10.1007/s11468-017-0514-4

[23] Chen, Fang-Chung, et al. "Plasmonic-enhanced polymer photovoltaic devices incorporating solution-processable metal nanoparticles." Applied Physics Letters 95.1 (2009): 182. https://doi.org/10.1063/1.3174914

[24] Yoon, Woo-Jun, et al. "Plasmon-enhanced optical absorption and photocurrent in organic bulk heterojunction photovoltaic devices using self-assembled layer of silver nanoparticles." Solar Energy Materials and Solar Cells 94.2 (2010): 128-132. https://doi.org/10.1016/j.solmat.2009.08.006

[25] Kulkarni, Abhishek P., et al. "Plasmon-enhanced charge carrier generation in organic photovoltaic films using silver nanoprisms." Nano letters 10.4 (2010): 1501-1505. https://doi.org/10.1021/nl100615e 
[26] Stratakis, Emmanuel, and Emmanuel Kymakis. "Nanoparticle-based plasmonic organic photovoltaic devices." Materials https://doi.org/10.1016/j.mattod.2013.04.006

Today $16.4 \quad$ (2013): 133-146.

[27] Diukman, Iddo, et al. "Controlling absorption enhancement in organic photovoltaic cells by patterning $\mathrm{Au}$ nano disks within the active layer." Optics express 19.101 (2011): A64-A71. https://doi.org/10.1364/OE.19.000A64

[28] Stranks, Samuel D., et al. "Electron-hole diffusion lengths exceeding 1 micrometer in an organometal trihalide perovskite absorber." Science 342.6156 (2013): 341-344. https://doi.org/10.1126/science.1243982

[29] Kambhampati, Patanjali. "Hot exciton relaxation dynamics in semiconductor quantum dots: radiationless transitions on the nanoscale." The Journal of Physical Chemistry C 115.45 (2011): 2208922109. https://doi.org/10.1021/jp2058673

[30] Ohkita, Hideo, et al. "Charge carrier formation in polythiophene/fullerene blend films studied by transient absorption spectroscopy." Journal of the American Chemical Society 130.10 (2008): 30303042. https://doi.org/10.1021/ja076568q

[31] Piralaee, Mina, A. Asgari, and V. Siahpoush. "Plasmonic properties of spheroid silicon-silver nanoshells in prolate and oblate forms." Optik 172 (2018): 1064-1068. https://doi.org/10.1016/j.ijleo.2018.07.131

[32] Krebs, Frederik C., et al. "Freely available OPV—-the fast way to progress." Energy Technology 1.7 (2013): 378-381. https://doi.org/10.1002/ente.201300057

[33] Daneshfar, Nader. "The Study of Scattering-to-absorption Ratio in Plasmonic Nanoparticles for Photovoltaic Cells and Sensor Applications." Plasmonics (2021): 1-7. https://doi.org/10.1007/s11468021-01464-z

[34] Pathak, Nilesh Kumar, P. Senthil Kumar, and R. P. Sharma. "Plasmonic perovskite solar cells utilizing noble metal-metal oxide hybrid nanoparticles." Noble Metal-Metal Oxide Hybrid Nanoparticles. Woodhead Publishing, 2019. 487- 498. https://doi.org/10.1016/B978-0-12-814134-2.00022-X

[35] Catchpole, KR and, and Albert Polman. "Plasmonic solar cells." Optics express 16.26 (2008): 21793- 21800. https://doi.org/10.1364/OE.16.021793

[36] Matheu, P., et al. "Metal and dielectric nanoparticle scattering for improved optical absorption in photovoltaic devices." Applied physics letters 93.11 (2008): 113108. https://doi.org/10.1063/1.2957980

[37] Mohan Jain, S. "Mutation-assisted breeding for improving ornamental plants." XXII International Eucarpia Symposium, Section Ornamentals, Breeding for Beauty 714. 2006. https://doi.org/10.17660/ActaHortic.2006.714.10

[38] Van Dijk, Jan AGM. "Digital divide research, achievements and shortcomings." Poetics 34.4-5 (2006): 221- 235. https://doi.org/10.1016/j.poetic.2006.05.004 
[39] Rai, Prabhakar. "Plasmonic noble metal@ metal oxide core-shell nanoparticles for dye-sensitized solar cell applications." Sustainable energy \& fuels 3.1 (2019): 63-91. https://doi.org/10.1039/C8SE00336J

[40] Gu, Qiongchan, et al. "Plasmon enhanced perovskite-metallic photodetectors." Materials \& Design 198 (2021): 109374. https://doi.org/10.1016/j.matdes.2020.109374

[41] Peng, Jinqing, Lin Lu, and Hongxing Yang. "Review on life cycle assessment of energy payback and greenhouse gas emission of solar photovoltaic systems." Renewable and sustainable energy reviews 19 (2013): 255-274. https://doi.org/10.1016/j.rser.2012.11.035

[42] Yee, Kane. "Numerical solution of initial boundary value problems involving Maxwell's equations in isotropic media." IEEE Transactions on antennas and propagation 14.3 (1966): 302-307. https://doi.org/10.1109/TAP.1966.1138693

[43] Atwater, Harry A., and Albert Polman. "Plasmonics for improved photovoltaic devices." Materials for sustainable energy: A collection of peer-reviewed research and review articles from nature publishing group (2011): 1-11. https://doi.org/10.1142/9789814317665_0001

[44] Kitai, Adrian. "Principles of solar cells, LEDs and diodes." Departments of Engineering Physics and Materials Science and Engineering, McMaster University, Hamilton (2011). https://doi.org/10.1002/9781119974543

[45] Huo, Yong, Hao-Li Zhang, and Xiaowei Zhan. "Nonfullerene all-small-molecule organic solar cells." ACS Energy Letters 4.6 (2019): 1241-1250. https://doi.org/10.1021/acsenergylett.9b00528

[46] Fan, Baobing, et al. "Achieving over 16\% efficiency for single-junction organic solar cells." Science China Chemistry 62.6 (2019): 746-752. https://doi.org/10.1007/s11426-019-9457-5

[47] An, Ning, et al. "Solution-Processed Organic Solar Cells with High Open-Circuit Voltage of 1.3 V and Low Non-Radiative Voltage Loss of 0.16 V." Advanced Materials 32.39 (2020): 2002122. https://doi.org/10.1002/adma.202002122

[48] Zhang, Yu, et al. "2D black phosphorus for energy storage and thermoelectric applications." Small 13.28 (2017): 1700661. https://doi.org/10.1002/smll.201700661

[49] Brabec, Christoph Joseph, et al., eds. Organic photovoltaics: concepts and realization. Vol. 60. Springer Science \& Business Media, 2003.

[50] Thompson, Barry C., and Jean MJ Fréchet. "Polymer-fullerene composite solar cells." Angewandte chemie international edition 47.1 https://doi.org/10.1002/anie.200702506

[51] Lightsource, Stanford Synchrotron Radiation. "Stable Solvent for Solution-based Electrical Doping of Semiconducting Polymer Films and Its Application to Organic Solar Cells." (2018). https://doi.org/10.1039/C8EE00811F

[52] Abass, Aimi, et al. "Dual-interface gratings for broadband absorption enhancement in thin-film solar cells." Physical Review B 85.11 (2012): 115449. https://doi.org/10.1103/PhysRevB.85.115449 
[53] Venugopal, N., et al. "Titanium nitride as light trapping plasmonic material in silicon solar cell." Optical Materials 72 (2017): 397-402. https://doi.org/10.1016/j.optmat.2017.06.035

[54] Samajdar, D. P. "Performance enhancement of Nanopyramid based Si hybrid solar cells utilizing the plasmonic properties of oxide coated Metal Nanoparticles." Optical Materials 107 (2020): 110166. https://doi.org/10.1016/j.optmat.2020.110166

[55] N'Konou, Kekeli, and Philippe Torchio. "Optical Absorption Modeling of Plasmonic Organic Solar Cells Embedding Ag-SiO2 Core-Shell Nanoparticles." Noble Metal-Metal Oxide Hybrid Nanoparticles. Woodhead Publishing, 2019. 265-282. https://doi.org/10.1016/B978-0-12-814134-2.00013-9

[56] Kluczyk, K., et al. "On modeling of plasmon-induced enhancement of the efficiency of solar cells modified by metallic https://doi.org/10.3390/nano9010003

[57] Qu, Di, et al. "Mechanism of optical absorption enhancement in thin film organic solar cells with plasmonic metal nanoparticles." Optics Express 19.24 (2011): 24795-24803. https://doi.org/10.1364/OE.19.024795

[58] Gunnarsson, L., et al. "Interparticle coupling effects in nanofabricated substrates for surfaceenhanced Raman scattering." Applied Physics Letters 78.6 (2001): 802-804. https://doi.org/10.1063/1.1344225

[59] Lu, Luyao, et al. "Cooperative plasmonic effect of $\mathrm{Ag}$ and $\mathrm{Au}$ nanoparticles on enhancing performance of polymer solar cells." Nano letters 13.1 (2013): 59-64. https://doi.org/10.1021/nl3034398

[60] Xu, Hongxing, and Mikael Käll. "Modeling the optical response of nanoparticle-based surface plasmon resonance sensors." Sensors and Actuators B: Chemical87.2 (2002): 244-249. https://doi.org/10.1016/S0925-4005(02)00243-5 